$$
\text { المجلة العراقية لعلوم الأرض- المجلدم- العددr- تثرين الثاني ^... }
$$

الطباقية البالينولوجية والبيئة القديمة للجزء العلوي من تكوين عكاس

في بئر KH5 / 1 في الصحراء الغربية العراقية

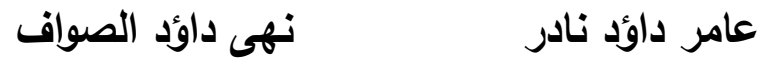

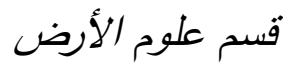

$$
\begin{aligned}
& \text { كلية العلوم }
\end{aligned}
$$

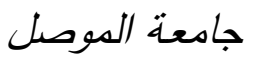

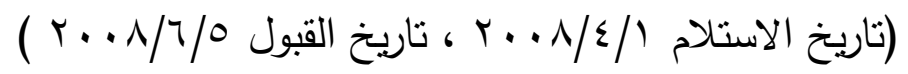

الملخص

تمت دراسـة حشود البـالينولات المحررة مـن ( ؟ ا ) نموذجاً من المقطع الطباقي المحصور بين

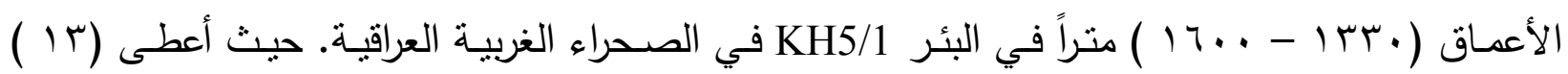

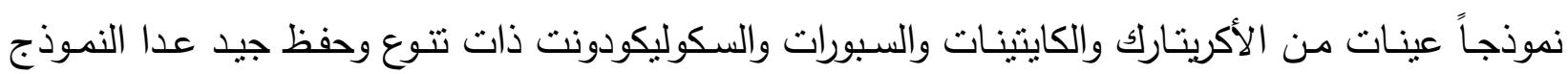

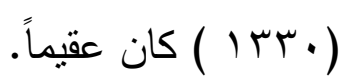

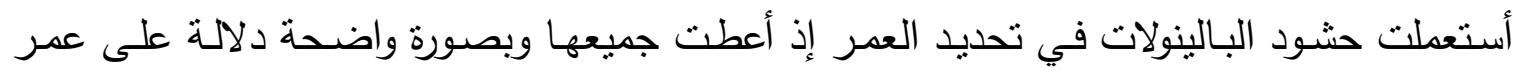
السايلوري العلوي ( Ludlow ) للمقطع الطباقي المدروس وذللك من خلال مقارنتها مع مناطق مختلفة من

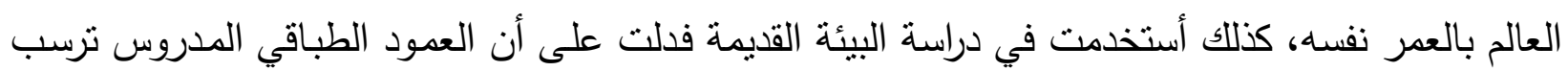
في بيئة قرب ساحلية.

\title{
Palynostratigraphy and Paleoecology of the Upper Part of Akkas Formation in Borehole KH5/1in the Western Iraqi Desert
}

\author{
Amer D. Nader Noha D. Al-Sawaf \\ Department of Geology \\ College of Science \\ Mosul University
}

\begin{abstract}
Fourteen sample from the stratigraphic interval $(1330-1600 \mathrm{~m}$.) in the borehole KH5/1 were studied palynoogically. The samples yielded well preserved and diversified palynomorphs except one sample at depth $(1330 \mathrm{~m}$.) was barren.
\end{abstract}




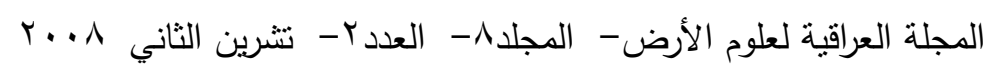

Identification of (54) species of acritarchs belong to (29) genera and (16) species of Chitinozoans belong to (9) genera, (16) species of miospores belong to (13) genera and (6) species of Scolecodont belong to (4) genera was the conclusion of this study.

Comparision with similar palynomorphs in different parts of the worlds indicates clearly Upper Silurian (Ludlow) age for the samples studied. The Palynomorphs and especially the acritarchs reveal that the studied section was deposited in near shore environment.

\section{المقدمة}

اشتملت الدراسـة الحالية على جزء من العدود الطباقي في بئر ( / KH5 ) وهو أحد الآبار الكثيرة التي حفرت في الصحراء الغربية العراقية عام (1919) لإغراض التنقيب الهيدروجيوكيمائي والهيدرولوجي، لقد حفر البئر (KH5/1 ) في منخفض الكعرة في الصحراء الغربية العراقية. إن الصحراء الغربية أرض منبسطة وذات ميل معتدل تمتد من الحدود الأردنية إلى نهر الفرات، ويعد منخفض الكعـرة الذي حفر بـه البئر (KH5/1) أهم صـفة جيومورفولوجيـة في الصـحراء الغربيـة العراقيـة.

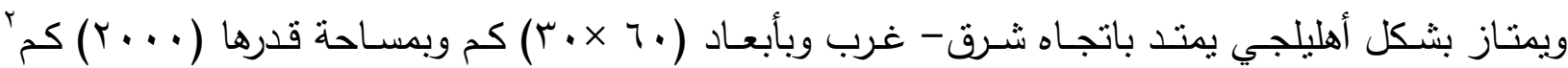
تقريباً. ويظهر في المنخفض عدد من الوديان الصغيرة من كل الجوانب إلا أن هناك مخرجا واحد فقط إلى نهر الفرات من خلال وادي حلقم المؤدي إلى وادي الرتكة (Al-Hadidy, 2001). ويبعد البئر حوالي ( ( ) كم إلى الثمال من مدينة الرطبة بنظر الثكل رقم (1). وقد أخترق البئر الصـور الرسوبية إلى عمق ( . . 7 ( ) متراً، بدأ من نكوين الكعرة والذي يعد أقدم الوحدات الصخرية المنكثفة على السطح في الصحراء

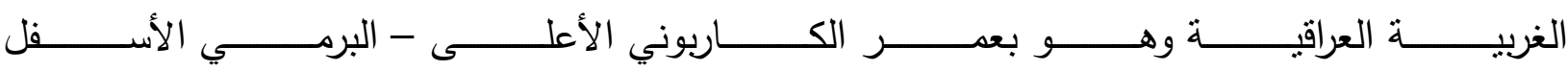
.(Nader et al., 1993)

أمسا أقدم التكوينـات في البئر فهو تكوين عكاس ( Akkas Formation) الذي أطلاق عليـه الجيولوجيون اليوغسلاف مع نكوين بيرسبكي وأورا وهارور تسمية تكوين الصوفي ( أي أطلق تكوين الصوفي

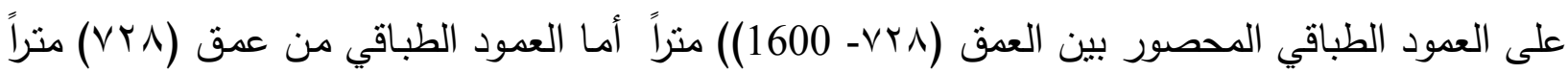
وإلى السطح فهو يمثل تكوين الكعرة.

لقد قسم (Al-Hadidy, 2001) العمود الطباقي في بئر ( KH5/l ) في دراسته الرسوبية للبئر إلى التكاوين التالية إعتماداً على مكافئاتها السطحية وهي تكوين بيرسبيكي ( • ع ا - . . ب ( ) متراً وتكوين أورا

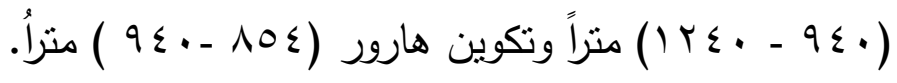

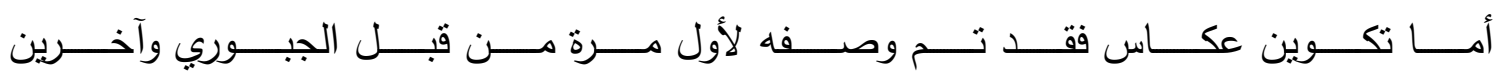
(Al-Jubbori et al., 1997) في العراق مـن البئر (KH5/1) من العمق (1300-1600) متراً ليمثل الصخور السجيلية الغرينية ذات اللون الرصاصي والسوداء المخضرة والحاوية على شرائط من الحجر الرملي 


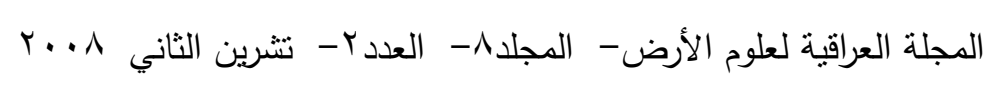

تهدف الدراسـة الحالية الى تحديد عمر العمود الطباقي العائد إلى تكوين عكاس والمحصور بين

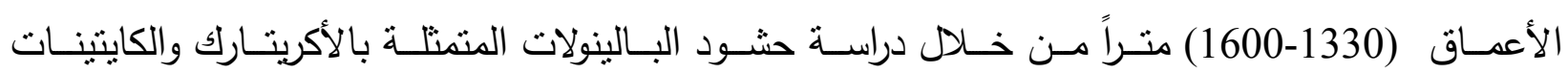
والمايوسبورات والسكوليكودونت الموجودة ضمن النماذج. إستتاج البيئة الترسيبية للجزء العلوي لتكوين عكاس من خلال دراسة حشود البالينولات.

\section{النماذج}

لقد أخذت نــاذج الدراسـة لجزءء مـن العمود الطباقي للبئر (KH5/1) والمحصـور بين الأعمـاق

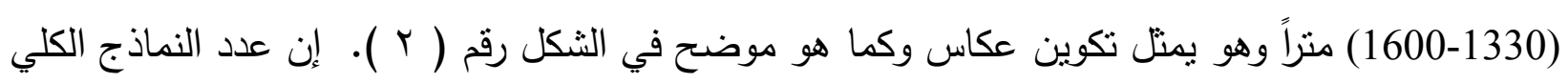

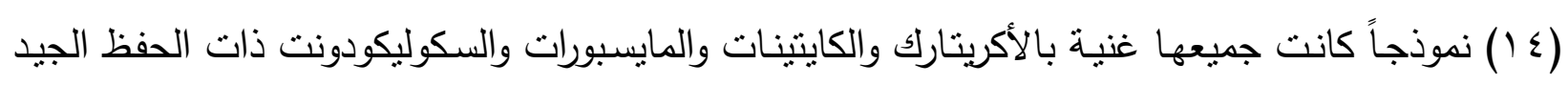
والتتوع الكبير عدا نموذج واحد هو النموذج ( 1330 ) . ولقد حُصل على نمـاذج الدراسة من المختبرات

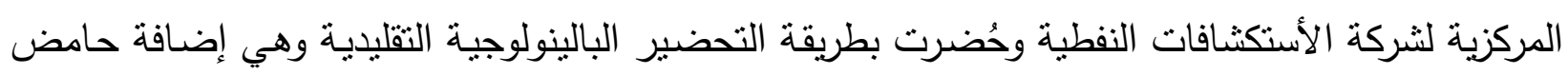

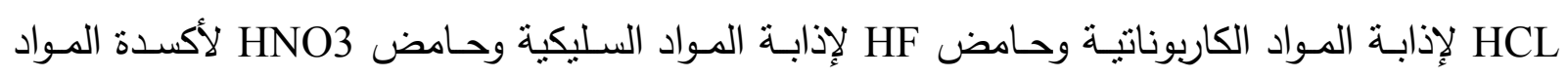

العضوية.

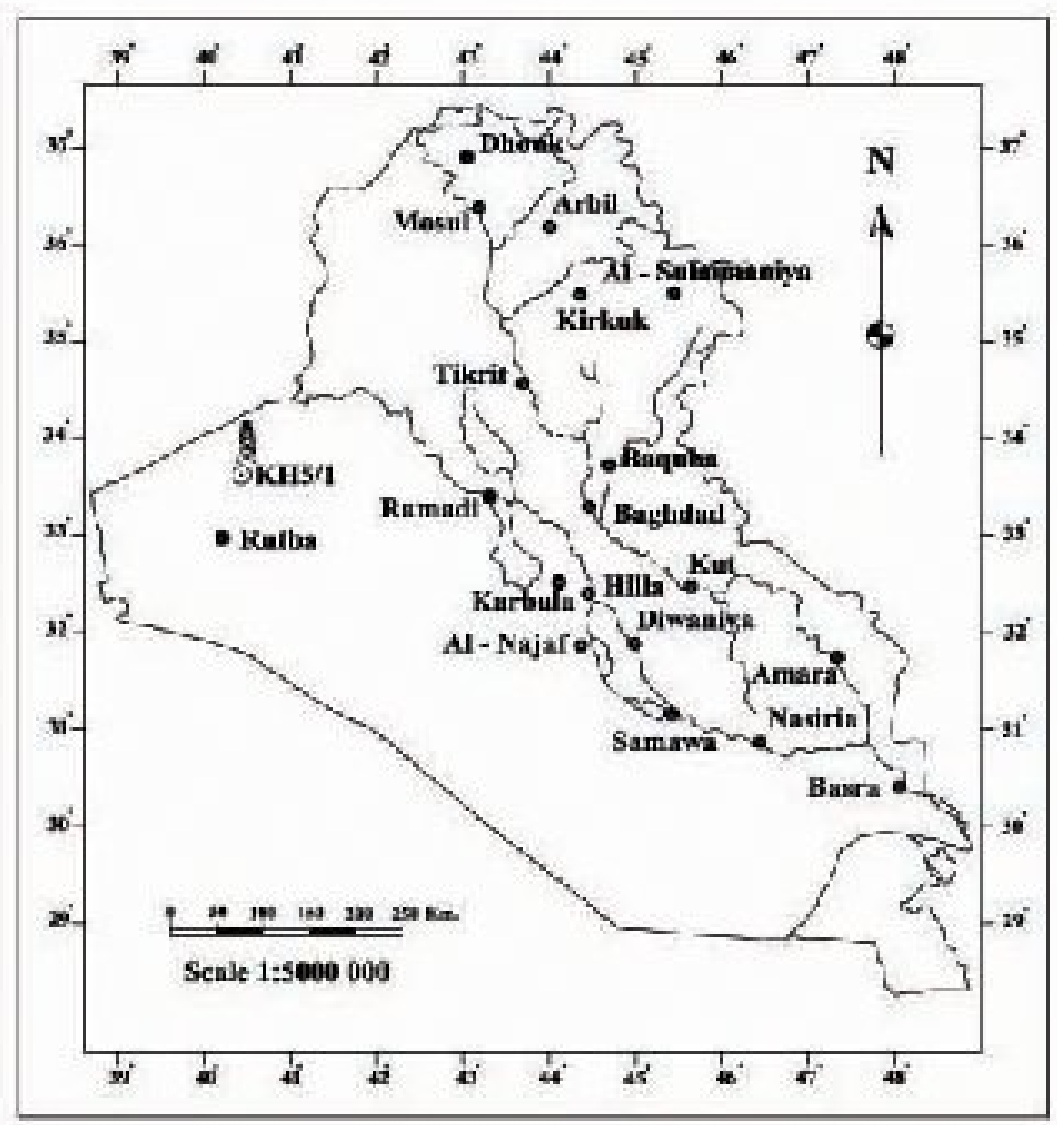

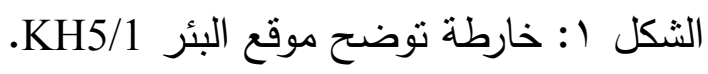




\begin{tabular}{|c|c|c|c|c|c|c|c|}
\hline 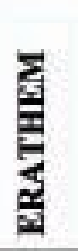 & $\frac{\sum}{\sqrt{2}}$ & $\frac{\text { 音 }}{\frac{1}{\sigma}}$ & 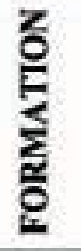 & 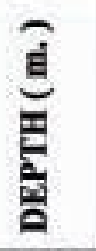 & 选 & LTTHOLOGY & LEGEND \\
\hline $\begin{array}{l}0 \\
-1 \\
0 \\
N \\
0 \\
4 \\
4 \\
4 \\
4 \\
4\end{array}$ & 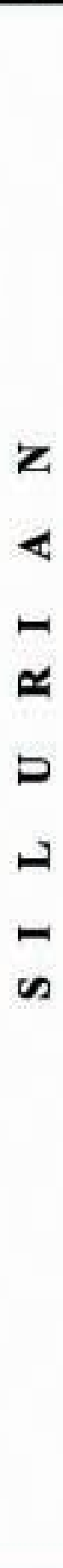 & $\begin{array}{l}\simeq \\
\text { A } \\
A \\
\text { A } \\
\end{array}$ & $\begin{array}{l}\infty \\
4 \\
\longleftarrow \\
4\end{array}$ & $\begin{array}{l}1300- \\
1310- \\
1320 \\
1330 \\
1340- \\
1350- \\
1360- \\
1370- \\
1380- \\
1390 \\
1400- \\
1410 \\
1420 \\
1430 \\
1440 \\
1450 \\
1460 \\
1470 \\
1480 \\
1490 \\
1500 \\
1510 \\
1520 \\
1530 \\
1540 \\
1550 \\
1560 \\
1570 \\
1580 \\
1590 \\
1600 \\
\end{array}$ & $\begin{array}{l} \\
\\
1522- \\
1528- \\
\\
19515- \\
1564- \\
1567- \\
1500- \\
1501.5- \\
1600-\end{array}$ & ( & Claystone \\
\hline
\end{tabular}

الثكل r ب: العمود الطباقي المدروس في البئر KH5/1 


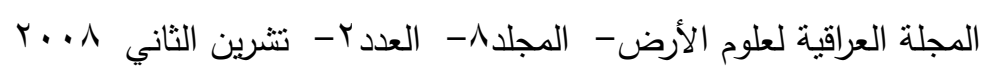

الطباقية الحياتية وتحديد العمر

أوضحت النماذج المدروسة مجاميع من الأكريتارك توزعت على (؟0) نوعاً تعود إلى (Y9) جنساً و

(7 ( ) نوعاً من الكايتينات تعود إلى (9) أجناس و (7 ( ) نوعاً من حشود السبورات تعود إلى (T I) جنساً.

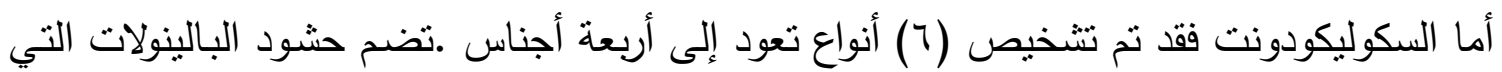

شخصت في هذه الدراسة أنواعا ذات دلالات واضحة على عصر السايلوري وتحديداً الجزء الأعلى منه عمر

- : وهذه الأنواع من الأكريتارك هي - Ludfordian

Cymbosphaeridium bikidum, Hapsidopalla sannemannii, Leiosphaeridia laevigata, L. major, Tasmanites aveloni, Leiofusa algerensis, L. elenae , L. estrecha, Eupoikilofusa striatifera, Deflandrastum colonnae, D. millepiedi, Domasia limaciforme, Dictyotidium biscutulatum ,D. coarcatum, Neoveryhachium carminae, Onondagella deunffii, Ovina desrtica.

إذ أن ظهور الأنواع : (lla sannemannii. Leoniella craminae, Onondagella deunffi, Ovina desrtica, Hapsidopalla sannemannii.

مع أستمرار ظهور أنواع الجنس Cymbosphaeridium ووصسول النوع Oeoveryhachium carminae نطاق ذروته يعد دليلاً على عمر السايلوري العلوي ( Ludlovian). كذلك فإن اعتمادنا في تحديد عمر هذه النماذج لم يقتصر على الأنواع الدالة من الأكريتارك بل لقد اعتمدنا على أدلة أخرى من السبورات والكايتينات إذ ظهرت سبورات السايلوري العلوي بصورة واضحة والتي يمكن تميزها بسهولة حيث غالباً ما تكون سبورات ثلاثيـة الفتحة تحوي عل تثخن على حافة السبور مثل جـس (Ambitisportites) وقـد يكـون التشخن علـى شـكل طـوق قـرب حافـة السـبور مثـل جـس كذللك السبورات الحاويـة على أضـلاع شعاعية على الوجه الأدنى للسبور مثل (Archaezonotriletes )

جنس(Emphanisporites).

ولقد ظهر لدينا العديد من السبورات الدالة المستخدمة عالمياً لتحديد عمر السايلوري العلوي منل : Retusotriletes warringtonii, R.minor, Emphanisporites annulatus, E.neglectus, Apiculiretusispora spicula, Ambitisporites avitus, A.dilutus, Archaeozontriletes chulus.

Aratrisporites saharaensis, Cyclogranisporites palaeophytus,

$$
\text { وبداية ظهور الأنواع : }
$$

Vallatisporites microspinosus.

$$
\text { هي من الأدلة الواضحة على عمر السايلوري الأعلى. }
$$

ولقد أظهرت حشـود الكايتينـات في هذه الدراسـة أيضـاً أدلـة واضـحة على عمـر السـايلوري العلـوي

$$
\text { حيث أن أنواع الكايتينات التي شخصت في هذه الدراسة تعود إلى النطاق الكايتيني: }
$$

Nestor, 1990 ) المثبت عند Sphaerochitina sphaerocephala (Chitinozoan biozone )

و Laufeld, 1974 ) في المنطقة الأسكندنافية والبلطيقية ( Baltoscandinavian Zone) والذي أستعمل

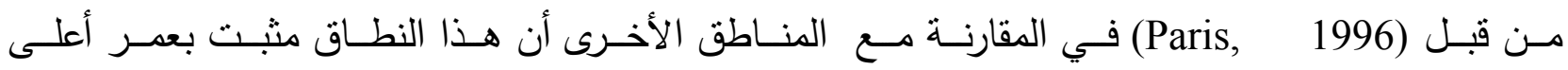




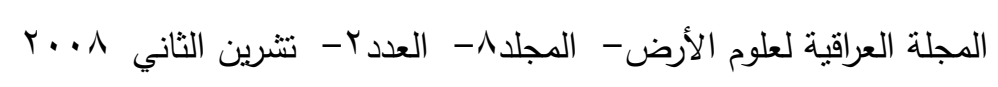

السايلوري أي ( Uppermost Ludlow) كما أنه مثبت بعمر ( Upper Ludlow ) في بريطانيا من قبل .(Aldrige et al., 1979; 1981) أن الكايتين النطاقي Sphaerochitina sphaerocephala ظهر في هذه الدراسة مترافقاً مـع أنواع

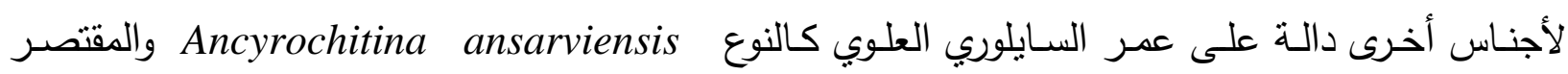
تواجده في السايلوري العلوي فقط والنوع Ancyrochitina pachyderma والدقتصر نواجده في شمال قارة كوندوانا والذي ظهر في هذه الدراسة بدل بشكل واضح على عمر السايلوري العلوي حيث أنه لم يثبت تواجده

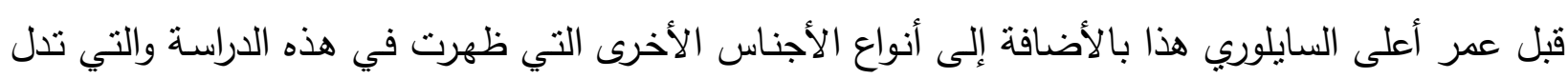

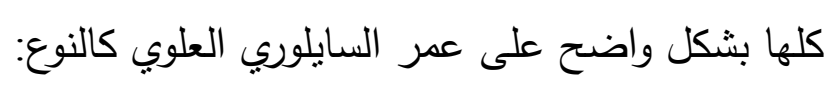

Linochitina hedei, Clathrochitina fritza, Lagenochitina elegans.

أما السكوليكودونت التي ظهرت لدينا في هذه الدراسة دلت على عمر السايلوري العلوي ( Ludlovian)

Arthrogenys spinys, Nereigenys paucislantis.

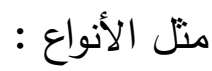

حيث أعتبر (Al-Ameri, 1980) أن مدى هذين النوعين مقتصر على عمر (Ludlovian).

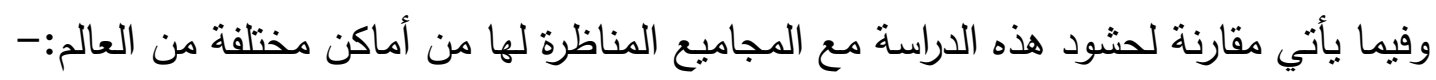

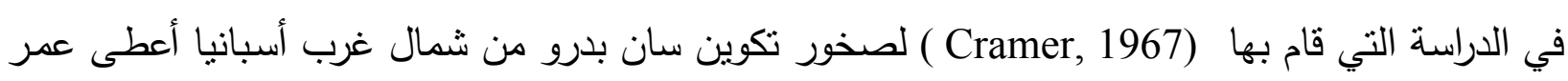
من الأكريتارك: معالوري العلوي ـ الديفوني السفلي) معتدا على الأنواع الدالة:-

Baltisphaeridium variopinnum, B.variopinnum lisum, Visbysphaera dilatispinosum, Geron guerillerus, Ellipsialetes laevigatus.

Ambitisporites avitus, Emphanisporites annulatus.

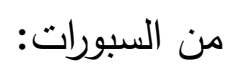

وكل هذه الأنواع كانت مشخصة في هذه الدراسة.

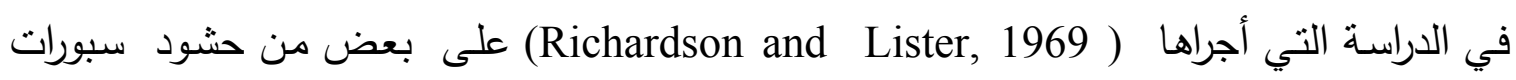

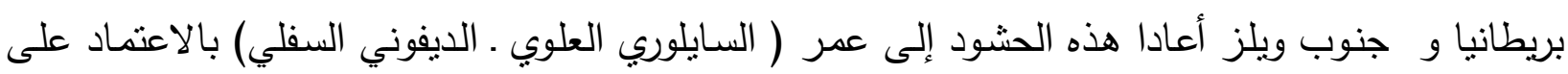
ظهور الأنواع التالية

Retusotriletes warringtonii, R. minor, Apiculiretusispora spicula, Ambitisporites avitus, A.dilutus, Archaeozonotriletes chulus.

وكل هذه الأنواع كانت متمثلة في دراستتا الحالية.

وفي الدراسة التي قام بها ( Lister, 1970) لصخور (السايلوري المتوسط ـ السايلوري الأعلى) من

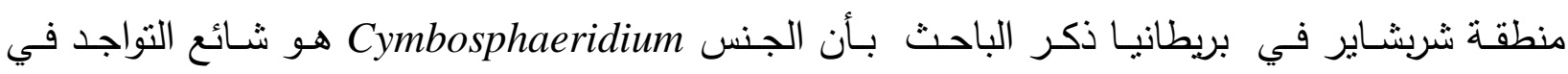

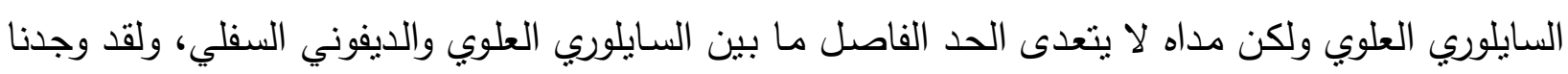

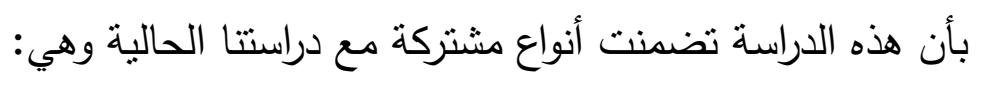




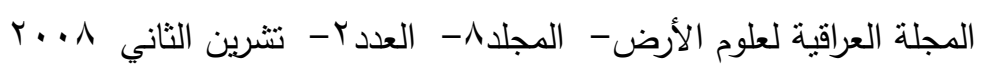

Cymbosphaeridium bikidum, Filisphaeridium williereae, Gorgonisphaeridium succinum, Micrhystridium stellatum, Visbysphaera dilatispinosum.

في الدراسـة التي قام بها كل من Richardson and Ioannides, 1973 ) للبالينولات من تكاوين

تانزوفت وأكاكوس من شمال أفريقيا بعدر السايلوري ظهرت الأنواع الآتية والتي تم تشخيصها من الدراسـة

Micrhystridium stellatum, Visbysphaera dilatispinosum, Neoveryhachium carminae, Leiofusa bernesgae L. estrecha, L. bernesgae

$$
\text { من السبورات : }
$$

Retusotriletes warringtonii, R.minor, Emphanisporites neglectus, Ambitisporites avitus, A.dilutus, Archaeozontriletes chulus.

وفي دراسة ( Aldridge et al., 1979 أعتمدوا على الكايتينات والسبورات والأكربتارك في تحديد

عمر السايلوري ، حيث اعتبروا ظهور أنواع الجنس Domasia يعتبر دليل على عمر (Ludlovian)، ولقد ظهرت أنواع مشتركة مع الدراسة الحالية من الأكريتارك

Cymbosphaeridium bikidium, Domasia limaciforme, Visbysphaera dilatispinosum, Leoniella craminae.

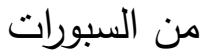

Apiculiretusispora specula, Archaezonotriletes chulus, Ambitisporites dilutus, Retusotriletes mino .

ومن دراسـة (A1 - Ameri, 1980) للطباقية الحياتية لحوض غداميس في ليبيا أعتمد الباحث في

$$
\text { تحديد (الجزء العلوي المتأخر) من السايلوري على بداية ظهور الأنواع : }
$$

Ambitisporites dilutus, Retusotriletes warringtonii, Archaeozonotriletes chulus, Geron guerillerus.

وهناك تشابه كبير مابين الحشود التي ظهرت في المنطقة المذكورة مع حشود منطقة دراستتا وممكن

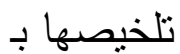

من الأكريتارك

Leiosphaeridia laevigata, L. major, Veryhachium trispinosum, Eupoikilofusa striatifera, Micrhystridium stellatum, Onondagella deunffi, Dictyotidium dictyotum, Neoveryhachium carminae, Geron guerillerus, Tasmanites aveloni.

Ambitisporites dilutus, Archaeozonotriletes chulus, Retusotriletes warringtonii.

ولقد ظهرت أيضاً حشود من البالينولات مشتركة مع بالينولات السايلوري من الدراسة التي قام بها )

لثمال ـ شرق ليبيا وهذه الأنواع هي 


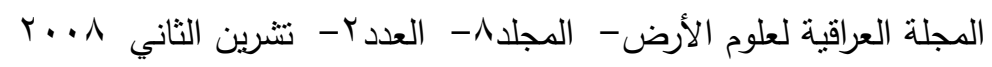

Eupoikilofusa striatifera, Veryhachium trispinosum,Neoveryhachium carminae, Dictyotidium dictyotum, Archaeozonotriletes chulus, Ambitisporites dilutus.

درس (Wicander, 1986) حشود الأكريتارك من تكوين هاركان من أوكلاهوما في الولايات المتحدة

Dictyotidium biscutulatum, D.coarctatum

$$
\text { الأمريكية حيث أعتمد على أنواع دالة في تحديد السايلوري العلوي مثل:- }
$$

و ذكر بـأن تواجد هذه الأنواع محصدر على عمر السـايلوري الأعلى وتختفي في نهايـة الديفوني

$$
\text { الأسفل وأعتمد في تحديد بداية الديفوني على أختفاء انواع الأجناس }
$$

Cymbosphaeridium, Deflandrastum, Leoniella, Visbysphaera.

ولقد ذكر أيضـاً بأن النوع Hapsidopalla sannemannii هو محصدر التواجد في (السـايلوري

$$
\begin{aligned}
& \text { العلوي ـ الديفوني السفلي). } \\
& \text { أما الأنواع }
\end{aligned}
$$

Veryhachium trispinosum, Veryhachium europaeum

ذات مدى جيولوجي طويل وإنتشـار جغرافي واسـع ولم يعتمد عليها في دراسته للطباقيـة الحياتية،

لذلك لم نعتمد نحن أيضا على هذه الانواع في دراستتا الحالية، والأنواع المشتركة في دراستتا مع هذه الدراسـة

Dicyotidium biscutulatum, D. coarcatum, Hapsidopalla sannemannii, Leiofusa estreacha, Veryhachium trispinosum V. europaeum.

وفي الدراسـة التي قام بها الباحثان Richardson and Rasul, 1990) لصخور من المملكة

المتحدة و ويلز والتي حددا عمرها (بالسايلوري العلوي) ظهرت نشابه لحشود الأكريتارك مع حشود الدراسـة الحالية وهي

Leoniella craminae, Onondagella deunffii, Gorgonisphaeridium succinum, Neoveryhachium carminae, Veryhachium trispinosum, Visbysphaera spp., Micrhstridium spp.

في الدراسـة التي قام بها (Molyneux et al., 1996 ) ذكروا بأن الجزء الأسفل من السـايلوري

Leoniella craminae تم تحديده في بريطانيا بالاعتمـاد على بداية ظهور النوع (Ludlow ) الـذي يعتبـر نــوع عـالمي و بدايــة ظهـوره مقصــور علـى هــذا العهـر فقــ، كـذللك ظهـور النـوع Onondagella deunffii الأسفل، كذلك أعتمدوا في تحديد السايلوري العلوي على ظهور نطاق

الذروة للنوع Neoveryhachium carminae والذي قد ظهر في نماذج هذه الدراسة بوفرة عالية، ولقد ظهرت أنواع مشتركة في الدراسة الآنفة الذكر مع دراستتا الحالية و هي:

Neoveryhachium carminae, Multiplicisphaeridium neahgae, Leiofusa algerensis, Geron guerillerus, Leoniella craminae, Onondagella deunfii, Deflandrastum spp., Visbysphaera spp. 


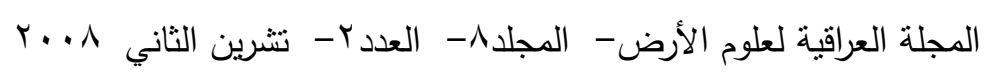

مهـا تقدم أعلاه يتضـح أن المقطع المدروس من تكوين عكاس يعود إلى حين(Epoch) السـايلوري العلوي وعمر Ludfordian.

\section{البيئة القديمة}

إن البالينولات التي ظهرت في هذه الدراسة تشتنل على البئل

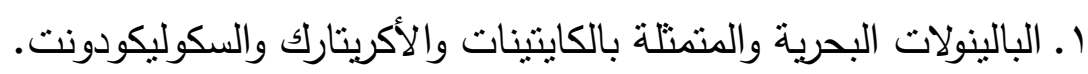
r. بالينولات اليابسة والمتمثلة بالسبورات.

أن نسب هذه البالينولات في النماذج المدروسة يعطي أدلة على بيئة الترسيب كذللك فإن ضمن خشورد

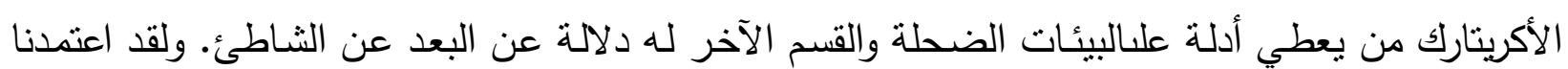

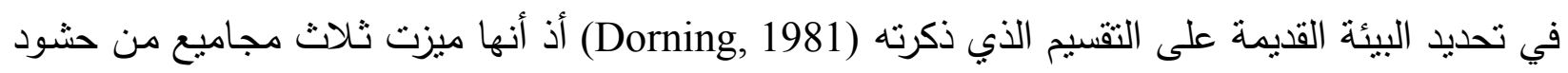
الأكريتارك في منطقة الجرف (Shelf) مع زيادة العمق والمسافة من خط الساحل وهذه المجاميع هي:

1 . حشود قرب الساحل ( Near shore assemblages ).

الذب يمثاز بالتتوع القليل إلى المعتدل (0 - 10 ) نوعاً في النموذج، وفيه تكون الأكريتارك الكروية

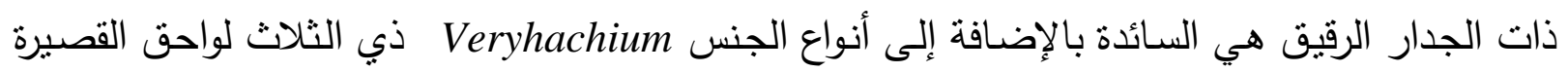

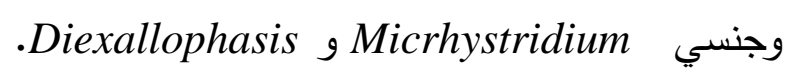

r. الحشود البعيدة عن الثاطئ( Ofshore assemblages)

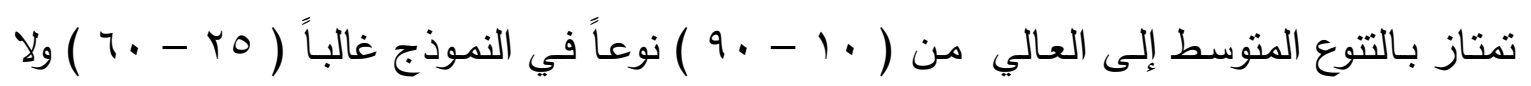
يوجد نوع من المصنفات هو الغالب.

r. حشود المياه العميقة ( Deep water assemblages )

التي تمتاز بالتتوع القليل إلى المعتدل ( r - 10 1 ) نوعاً في النموذج الواحد الأكريتارك الكروية تكون ذات جدار سميك أملس، والأجناس الأخرى تظهر بأعداد قليلة مثل Lophosphaeridium, Micrhystridium, Veryhachium.

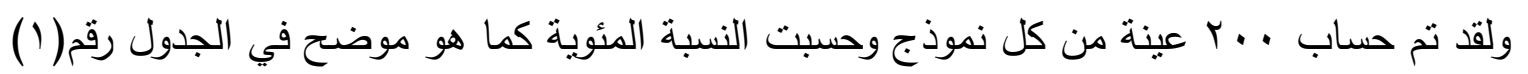

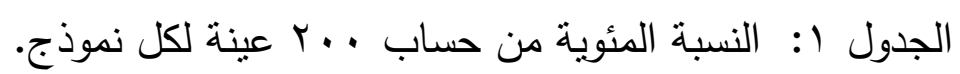

\section{Palynomorphs}




\begin{tabular}{|c|c|c|c|c|c|c|}
\hline Samples & السبورات & الأكريتارك & الأكريتارك & الأكريتارك & الأكريتارك ذات & $\begin{array}{c}\text { السكايتينات } \\
\text { السكوليكودونت }\end{array}$ \\
\hline . & $\cdots$ & $\cdots$ & $\ldots$ & $\cdots$ & $\cdots$ & $\cdots$ \\
\hline צr" & r.. & Ir.. & $V V .0$ & 7. &.$v$ & $\cdot . \wedge$ \\
\hline אזrו & $\leqslant \wedge$ & $0 .$. & $r q$ & 7.. & $\cdots$ & T.. \\
\hline 11599 & r.0 & $1 T .0$ & $\varepsilon \varepsilon \ldots$ & r. & $1 .$. & r \\
\hline س & .0 & r & $7 V .0$ & 7.0 & $\cdots$ & T.O \\
\hline $1 \leq \varepsilon r$ & $\varepsilon .0$ & 11.0 & rq & 9.0 & $\cdots$ & 1.0 \\
\hline lOY & Y.O & rA.0 & $0 \leqslant .0$ & 9.0 & 1.0 & r.0 \\
\hline lork & $\wedge .0$ & سT & $\varepsilon r$ & 11.0 & $\varepsilon .0$ & .0 \\
\hline 1001.0 & 11.0 & rA.0 & rq & 11 & r.0 & .01 \\
\hline 1075 & 10 & ro & r & iv & 1.0 & r.o \\
\hline $107 \mathrm{~V}$ & r.. & $r \wedge$ & or & $1 . .0$ & r.o & $\varepsilon$. \\
\hline $10 \mathrm{~V}$. & 1. & Y).0 & 7. & o. & Y.. & Y.. \\
\hline 1091.0 & 1.0 & rI & r & r. & 1.0 & .0 \\
\hline $17 \ldots$ & ^.. & $\varepsilon \vee .0$ & หч.. & $1 V .0$ & .0 & .0 \\
\hline
\end{tabular}

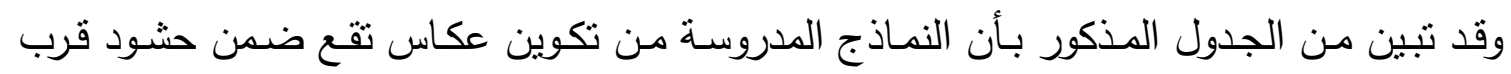

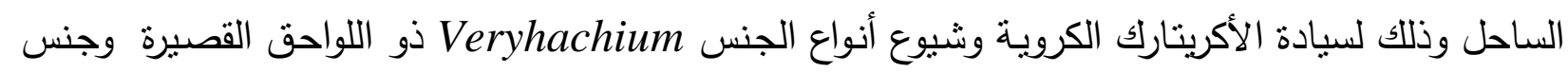

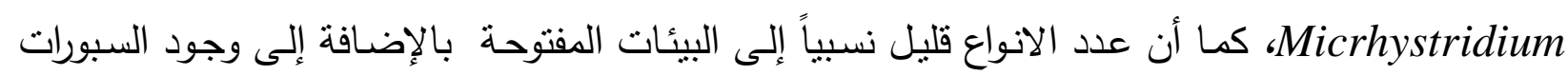
التي تدل على بيئة قرب الساحل.

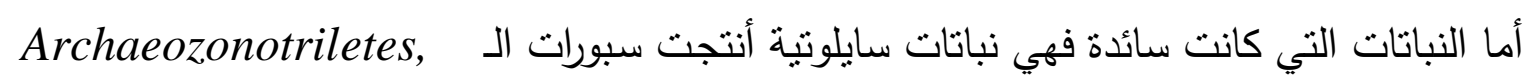
Ambitisporites

\section{| الإستنتاجات}

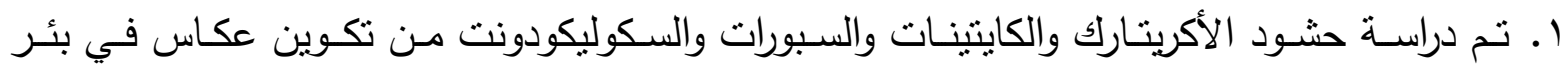

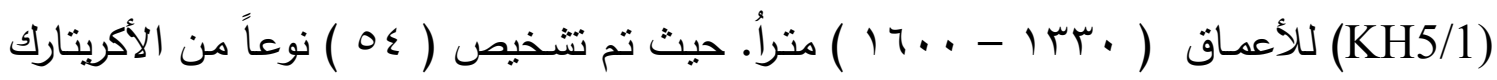

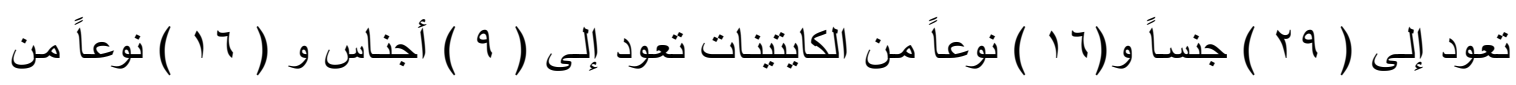




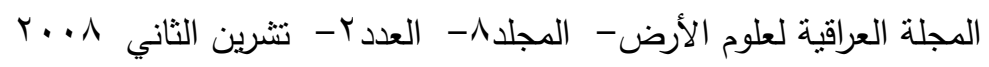

أسبورات تعود إلى ( T T ) جنساً. أما السكوليكودونت فقد تم تشخيص ( T ) أنواع تعود إلى (ع )

ץ. بالأعتماد على الحشود المشخصـة من الأكريتارك والكايتينات والسبورات والسكوليكودونت ومقارنتها

مع دراسات سابقة في منطقة الثرق الأوسط والمناطق المجاورة تم تحديد عمر المقطع المدروس من

تكوين عكاس للبئر (KH5/1 ) بالسايلوري العلوي (Ludlow).

ץ. أظهرت الحثـود التي تم تشخيصـها في الدراسـة الحسالية بأن البيئة القديمـة لتكوين عكاس تتمثنل

ببيئة قرب ساحلية.

\section{REFERENCES}

Al-Ameri, T.K., 1980. Palynology, Biostratigraphy and Palaeocology of Subsurface Mid- Palaeozoic Strata From the Ghadames Basin, Libya. Unpublised Ph.D. thesis, University of London, King's College.London.477p.

Aldridge, R.J.; Dorning, K.J.; Hill, P.J.; Richardson, J.B and Siveter, D.J., 1979. Microfossil Distribution in the Silurian of Britain and Ireland. The Caledonides of the British Isles Reviewed; Special Puplication of the Geological Society of London, pp. 433-438.

Aldridge, R.J.; Dorning, K.J. and Siveter, D.J., 1981. Distribution of Microfossil Groups Across the Wenlock Shelf of the Welsh Basin; in : NEALE, J.W. and BRASIER, M.D.(ed.); Microfossils from Recent and Fossil Shelf Seas Elis Horwood Ltd., pp.18-30.

Al-Hadidy, A.H., 2001. Sedimentary Facies and Depositional Environments of Late Paleozoic Successions (Devonian-Permian) in Iraq; Unpublised Ph.D. thesis, University of Mosul, Iraq. 159p.

Al-Jubbori, F.H.; Youkhana, A.K.; Al-Rubaii,M.A and Al-Samarrai, A.I., 1997. The Akkas Formation : A New Silurian lithostratigraphic Unit in Iraq; Oil Exploration Company, Baghdad.

Cramer, F.H., 1967. Palynomorphs from the Siluro- Devonian Boundary in NW Spain; Notasy Comunincationes Del Instituo. Geolo.y Minero De Espana, N.85, pp.71-82.

Dorning, K.J., 1981. Silurian Acritarch Distribution in the Ludlovian Shelf Sea of South Wales and the Welsh Borderland; in Neale, J.W. and Brasier, M.D. (ed), Microfossils From Recent and Fossil Shelf seas, Ellis Horwood Ltd. Chichester, pp.31-36.

HILI, P.J., PARIS, F. and RICHARDSON, J.B., 1985., Silurian palynomorphs. J., Micropaleontol.,Vol.4, pp.27-48.

Laufeld, S., 1974., Silurian Chitinozoa, Gotland, Sweeden; Fossils and Strata, pp.1-130.

Lister, T.R., 1970. The Acritarchs and Chitinozoa from the Wenlock and Ludlow Series of the Ludlow and Millichope areas, Shropshire; Palaeontogra. Soc.(Monogr.), pp.1-100.

Molyneux, S.G.; Leherisse, A. and Wicander, R., 1996., Chapter 16. Paleozoic phytoplankton; in:Jansonius, J. and McGregor, D.C.(ed.), Palynology: principles and 


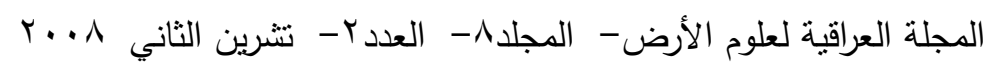

applications; American Association of Stratigraphic Palynologists Foundation Vol.2, pp.493 - 529.

Nader, A.D.; Khalaf, F.H. And Yousif, R.A., 1993., Palynology of the Upper part of the Ga'ara Formation in the Western Iraqi Desert; Mutah Jour. Res. Stud., Vol.8, pp.77-101.

Nestor, V., 1990. Silurian chitinozoans; in KALJO, D. and Nestor, H.(ed.), Field Meeting Estonia 1990. An Excursion Guidebook; Estonian Academy of Sceinces, Institute of Geology, pp.80-83.

Paris, F., 1996. Chapter 17. Chitinozoan Biostratigraphy and Palaeoecology; in: Jansonius,J. and McGregor, D.C.(ed.), Palynology :principles and applications; American Association of Stratigraphic Palynologists Foundation,Vol.2, pp.531- 552 .

Richardson, J.B. and Ioannides, N., 1973. Silurian Palynomorphs From the Tanezzuft and Acacus Formations, Tripolitania, North Africa; Micropaleontology, Vol.19, pp.275-307.

Richardson, J.B. and Lister. T.R., 1969. Upper Silurian and Lower Devonian Spores Assemblages From the Welsh Borderland and South Wales; Palaeontology, Vol.12, pp. 201-252.

Richardson, J.B. and Rasul, S.M., 1990. Palynofacies in a Late Silurian Regressive Sequence in the Welsh Borderland and Wales; Jour. of the Geol. Soc. London. pp. 675-686.

Wicander., E.R., 1986., Lower Devonian (Gedinnian) acritarchs from the Haraga Formation, Oklahoma, U.S.A; Rev. Palaeobotany and Palynology. pp.327-365. 


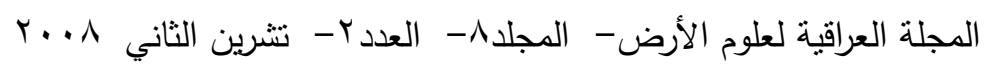

\section{PLATE - 1}

1. Cymbosphaeridium bikidum Lister 1970, 15671, L 1.5/132.2, Size $87.5 \mu \mathrm{m}$.

2. C. bikidum Lister 1970, 15671,L 0.8/128.5,Size $87.5 \mu \mathrm{m}$.

3. Hapsidopalla sannemannii (Deunff) Playford 1977, 16002,L 10.8/120, Size $42.5 \mu \mathrm{m}$.

4. Leoniella craminae Cramer $1964,13931, \mathrm{~L}$ 1.5/123.2,Size 87.5 $\mu \mathrm{m}$.

5. Leiosphaeridia laevigata Stockmans \& Willierie 1963,16002, L11.5/127.2, Size75 $\mu \mathrm{m}$.

6. L. major (Staplin) Downie \& Sarjent 1964,15701, L 12/125, Size77.5 $\mu \mathrm{m}$.

7. Tasmanites aveloni Somer 1953,15911,R 4.5/120.2,Size $72.5 \mu \mathrm{m}$.

8. Leiofusa algerensis Cramer 1970, 1442.32, R 9.2/128.5, Size $255 \mu \mathrm{m}$.

9. Leiofusa elenae Cramer 1964, 1567 1, L 3.4/123.9, Size 225 $\mu \mathrm{m}$.

10. L. estrecha Cramer 1964, 1567 1, R 1.8/119.8, Size175 $\mu \mathrm{m}$.

11. L. estrecha Cramer 1964, 1553.5 2, L 1.8/133.3, Size $325 \mu \mathrm{m}$.

12. Eupoikilofua striatifera (Cramer) Cramer 1970, 1567 2, R 9/120.5, Size $225 \mu \mathrm{m}$.

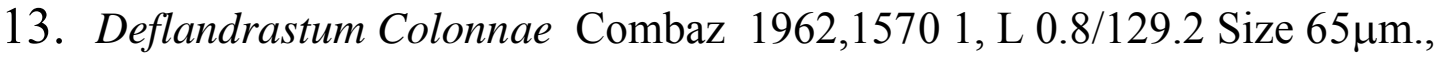

14. D. millepiedi Combaz 1962, 1570 1, L 0/125, Size $157.5 \mu \mathrm{m}$.

15. Domasia limaciforme(Stockmans \& Williere) Cramer 1970, 1600 1, R1/134.5, Size $267 \mu \mathrm{m}$.

16. Neoveryhachium carminae Cramer 1964,1600 1, L 4.5/125.5, Size $35 \mu \mathrm{m}$.

17. Onondagella sanpetrensis Cramer 1966, 1522 1, L 0.2/122, Size $50 \mu \mathrm{m}$.

18. Onondagella deunffi Cramer 1966, 1600 2, L 10.8/120.5, Size $75 \mu \mathrm{m}$.

19. Dictyotidium biscutulatum Kirjanov 1978, 1600 1, L 0.5/129, Size $45 \mu \mathrm{m}$.

20. D. biscutulatum Kirjanov 1978, 1393 1, L 1.5/130, Size $42.5 \mu \mathrm{m}$.

21. Dictyotidium coarctatum ( Kirjanov ) Colbath 1983, 1600 1, L 4/121, Size 37.5 $\mu \mathrm{m}$.

22. Ovina desrtica Cramer \& Diez 1977, 1528 1, L 9.7/129, Size $57.5 \mu \mathrm{m}$. 


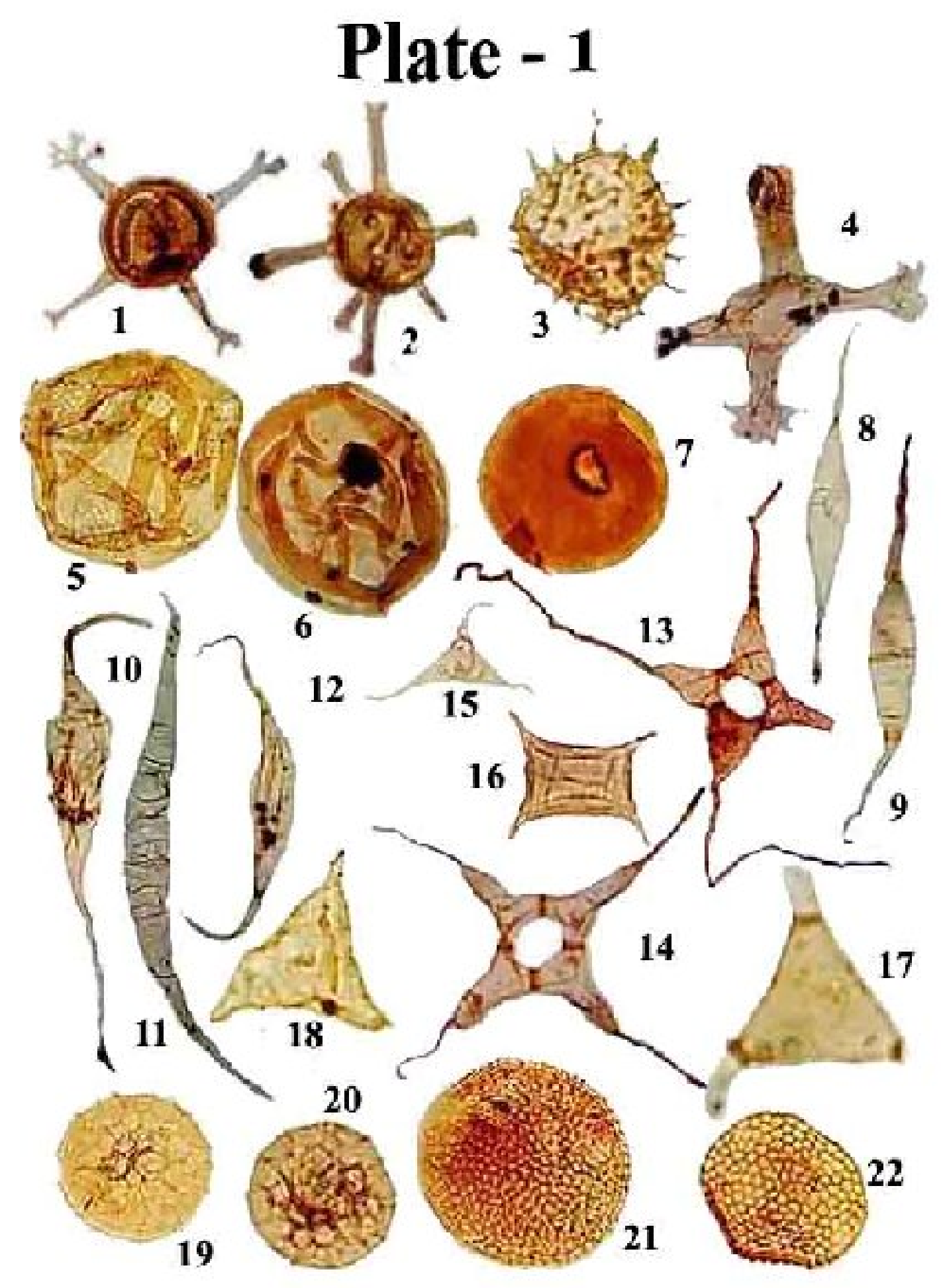




\section{PLATE - 2}

1. Ancyrochitina ancyrea (Eisenack) Laufeld 1971, 1570 ${ }^{1}$, 2 2.8/121.5, Size130 $\mu \mathrm{m}$.

2. A. ancyrea (Eisenack) Laufeld 1971, 15671, R 8.2/129.5, Size175 $\mu \mathrm{m}$.

3. A. ansarviensis Laufeld 1974, 15672, R 6.8/125.5, Size155 $\mu \mathrm{m}$.

4. Ancyrochitina longicornis Taugourdeau \& Jekhowski 1960,13932, L 3/130.5, Size115 $\mu \mathrm{m}$.

5. Ancyrochitina pachyderma Laufeld 1974, 14421, L 6.7/128.5, Size125 $\mu \mathrm{m}$.

6. A. pachyderma Laufeld 1974,13931, L 2.8/132, Size107.5 $\mu \mathrm{m}$.

7. Clathrochitina fritza Al-Ameri 1980, 15672, R 0.5/121.5,Size $165 \mu \mathrm{m}$.

8. C. fritza Al-Ameri 1980,15672, R 5.4/133, Size $142.5 \mu \mathrm{m}$.

9. Cyathochitina kuckersiana kuckersiana Eisenack 1955, 15701, L 4.8/129, Size $90 \mu \mathrm{m}$.

10. Gotlandochitina tabernaculifera Laufeld 1974,13931, L 3.5/131.2, Size155 $\mu \mathrm{m}$.

11. Lagenochitina elegans Beju \& Danet 1962, 13931, L 0.2/125, Size150 $\mu \mathrm{m}$.

12. L.elegans Beju \& Danet 1962, 13693, L 0.8/127.1, Size $137.5 \mu \mathrm{m}$.

13. Linochitina hedei Laufeld 1974, 14421, L 2.1/135, Size125 $\mu$ m.

14. L.hedei Laufeld 1974,15671,R 1.8/119, Size95 $\mu \mathrm{m}$.

15. L. hedei Laufeld $1974,15701, \mathrm{R} 0.2 / 126.5$, Size62.5 $\mu \mathrm{m}$.

16. Plectochitina craminae Cramer 1964, 15672, R 7/125, Size76 $\mu \mathrm{m}$.

17. Plectochitina pseudoagglotinans (Taugourdeau ) Cramer 1964,13931, R 7.8/131.8, Size105 $\mu \mathrm{m}$.

18. Sphaerochitina acanthifera Eisenack 1955, 15641, L 0.5/132.4, Size $205 \mu \mathrm{m}$.

19. S.acanthifera Eisenack 1955, 13693, L 0/129.7, Size $210 \mu \mathrm{m}$.

20. S.acanthifera Eisenack 1955, 15641, L 6/125, Size255 $\mu \mathrm{m}$.

21. Sphaerochitina sphaerocephala (Eisenack) Eisenack 1955. L 3/120.5, Size117.5 $\mu \mathrm{m}$. 


\section{Plate - 2}
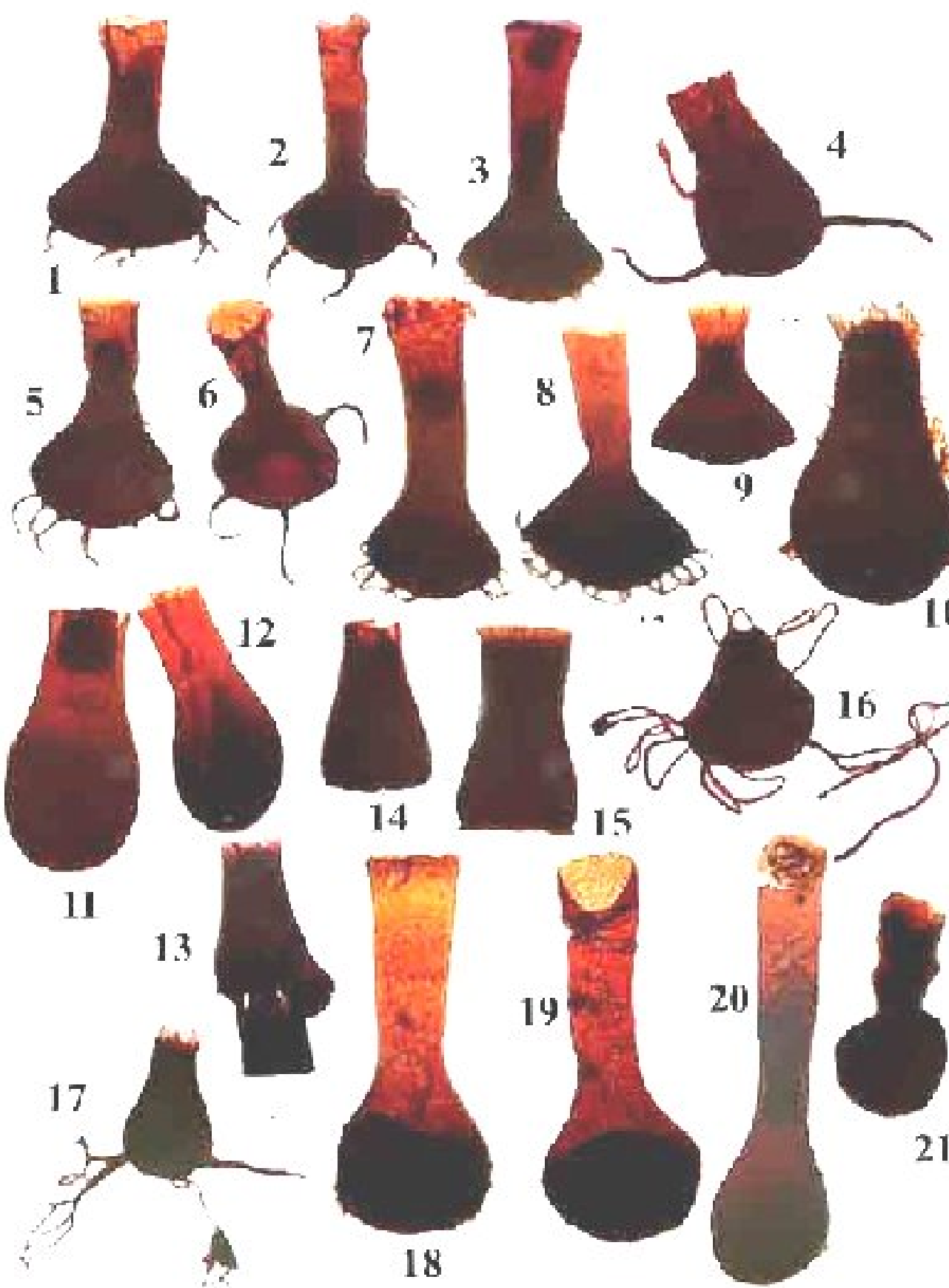

M
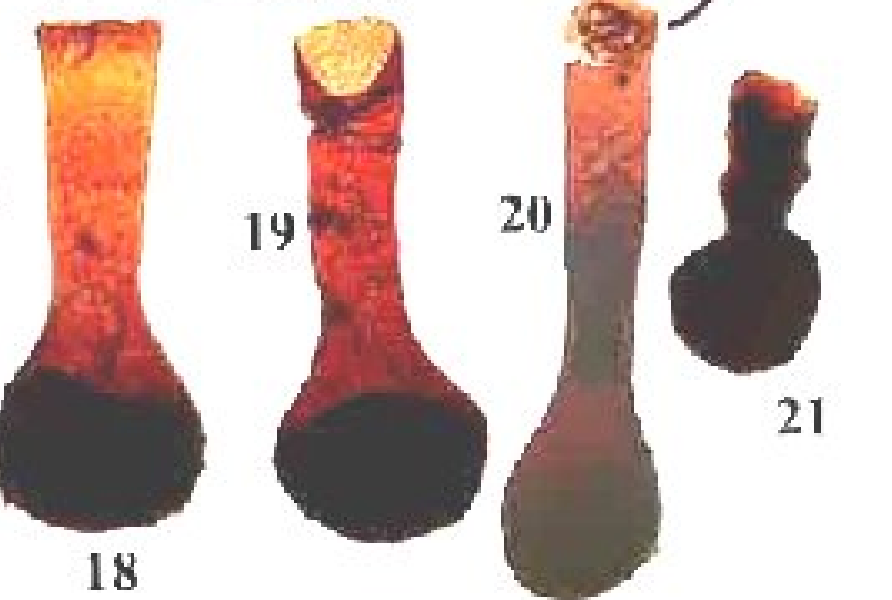


\section{PLATE - 3}

1.Retusotriletes minor Kedo 1963, 1600 ${ }^{1}$, R 0/129, Size $40 \mu \mathrm{m}$.

2. Retusotriletes warringtonii Richardson and Lister 1969, $1333^{2}$, R 3.5/125, Size $40 \mu \mathrm{m}$.

3. Emphanisporites annulatus McGregor 1961, 1567 , L 5/132.5, Size $57.5 \mu \mathrm{m}$.

4.E. neglectus Vigran 1964,1600², L 0.8/130 .5, Size $33.5 \mu \mathrm{m}$.

5.Ambitisporites avitus Hoffmeister 1959, 1553.5 ${ }^{1}$, L 1.2/131.7, Size $40 \mu \mathrm{m}$.

6.Ambitisporites dilutus (Hoffmeister) Richardson \&Lister 1969, 1570¹, R 7.5/128, Size $35.5 \mu \mathrm{m}$.

7. Archaeozonotriletes chulus ( Cramer ) Richardson and Lister 1969, 1600², L 11.5/127, Size $32.5 \mu \mathrm{m}$.

8.Leodicites falciformis ( Staufer ) Taugourdeau 1968, 1591.4.2/13, Size $87.5 \mu \mathrm{m}$.

9. Nereigenys paucislantis $\mathrm{Al}$ - Ameri $1980,1600^{\circ}, \mathrm{R} 2.1 / 130.5$, Size $87.5 \mu \mathrm{m}$.

10. Arthrogenys spinys Al- Ameri 1980, $1600^{\circ}, \mathrm{L} 4.2 / 118.5$, Size $87.5 \mu \mathrm{m}$.

11. Ildraites cf.howelli Eller 1936 in Al- Ameri 1980, 1591.5 , 1.5/122, Size $275 \mu \mathrm{m}$.

12. Ildraites howelli Eller 1936, $1600^{2}$, L 7/131.2, Size $155 \mu \mathrm{m}$. 


\section{Plate - 3}
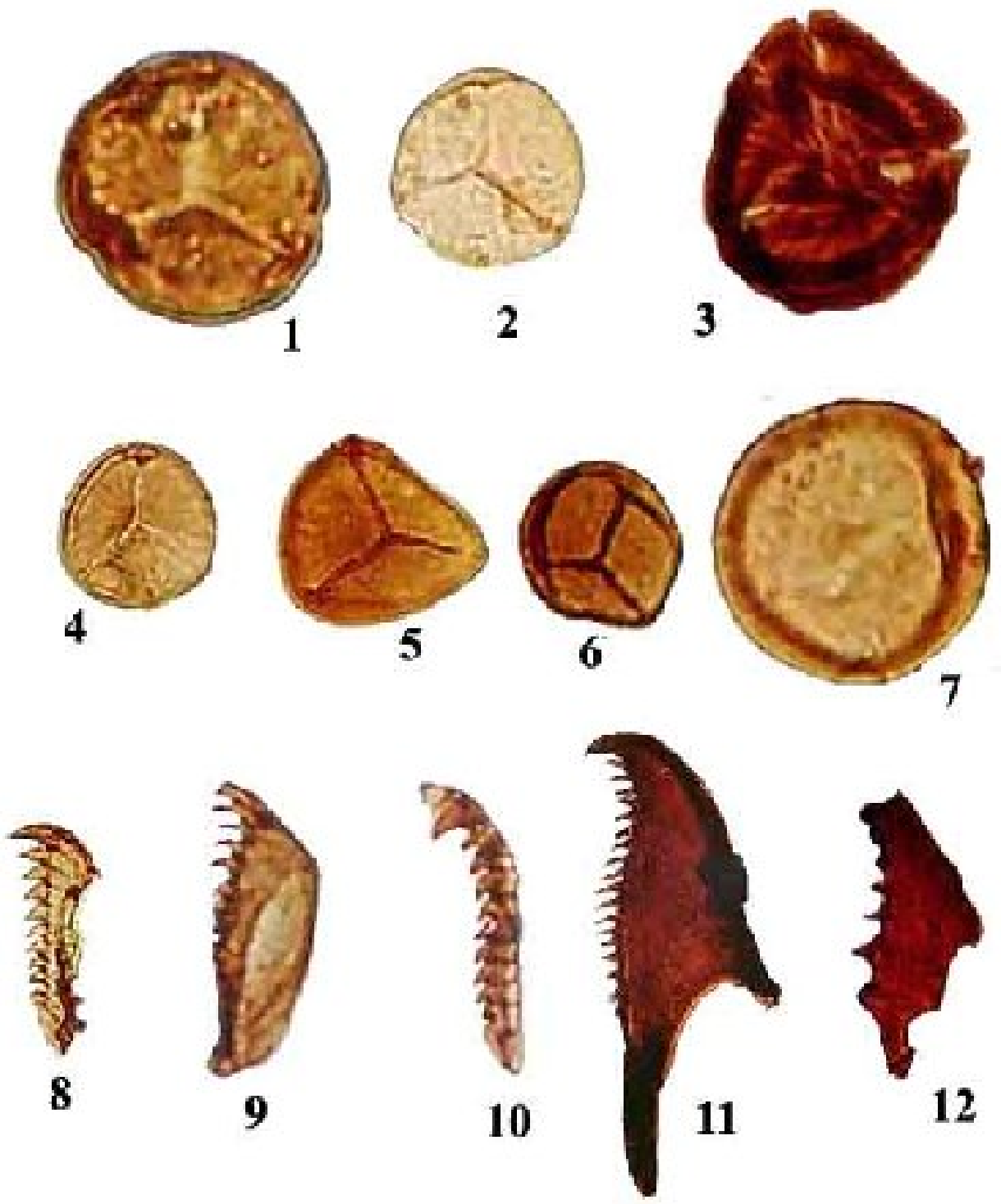